\title{
ENVELHECIMENTO ATIVO: UM PANORAMA DO INGRESSO DE IDOSOS NA UNIVERSIDADE
}

\author{
Silvia Virginia Coutinho Areosa ${ }^{1}$ \\ Cristiane Redin Freitas ${ }^{2}$ \\ Melissa Lampert ${ }^{3}$ \\ Cláudia Tirelli ${ }^{4}$
}

\section{Resumo}

Este estudo toma como base o contexto social presente na atualidade que destaca o envelhecimento populacional e a longevidade como desafios para a sociedade. Deste modo, seu objetivo principal é apresentar dados sobre a inserção de idosos em cursos de graduação na Universidade de Santa Cruz do Sul/RS. O aumento de idosos em cursos superiores mostra uma nova faceta do envelhecimento, marcando a possibilidade de um envelhecimento satisfatório com qualidade de vida. Para tanto, apresentam-se dados comparativos da inserção de idosos em cursos de graduação nos anos de 2004 e 2015. A ampliação da proporção de pessoas idosas em idade ativa que busca por uma nova formação, pode representar além da ocupação do tempo livre, a inserção no mercado de trabalho e uma nova perspectiva para esta fase da vida.

Palavras-chave: Envelhecimento Ativo; Universidade; Desenvolvimento Social

\footnotetext{
${ }^{1}$ Doutora em Serviço Social. UNISC, Universidade de Santa Cruz do Sul. Endereço: Av. Independência, 2293, Bairro Universitário, Santa Cruz do Sul, RS, Brasil. Endereço eletrônico: sareosa@unisc.br

${ }^{2}$ Doutora em Psicologia Social. UNISC, Universidade de Santa Cruz do Sul. Endereço: Av. Independência, 2293, Bairro Universitário, Santa Cruz do Sul, RS, Brasil. Endereço eletrônico: cristianefr@unisc.br

${ }^{3}$ Doutora em Medicina e Ciências da Saúde. UNISC, Universidade de Santa Cruz do Sul. Endereço: Av. Independência, 2293, Bairro Universitário, Santa Cruz do Sul, RS, Brasil. Endereço eletrônico: melissalampert@unisc.br

${ }^{4}$ Doutora em Sociologia. UNISC, Universidade de Santa Cruz do Sul. Endereço: Av. Independência, 2293, Bairro Universitário, Santa Cruz do Sul, RS, Brasil. Endereço eletrônico: ctirelli@unisc.br
} 


\section{INTRODUÇÃO}

Uma das grandes mudanças que podem ser observadas nos países em desenvolvimento, nas últimas décadas, refere-se a alteração do perfil etário de sua população. O Brasil, de acordo com os dados do Censo Demográfico 2010 (IBGE) acompanha esta tendência, demonstrando uma redução na taxa de fecundidade e, ao mesmo tempo, uma elevação da expectativa de vida da população, o que configura um aumento da população nas faixas etárias mais avançadas. De acordo com os dados do Censo 2010 (IBGE), as pessoas com mais de 60 anos representavam $11 \%$ da população total. Se observarmos os dados referentes ao Estado do Rio Grande do Sul, no qual se insere esta pesquisa, essas mudanças se mostraram ainda mais marcantes, pois este estado apresentou um acréscimo de mais de oito anos na expectativa de vida entre 1979 e 2010, passando de 67,8 para 75,9 anos, a segunda posição em longevidade entre todos os estados da federação. No RS, a população com mais de 60 anos representava 13,5\% da população total do estado em 2010. Segundo a projeção apresentada no documento RS 2030 (FEE, 2014), a partir de 2020 a quantidade de pessoas entre 15 e 64 anos vai começar a sofrer um processo de redução no estado, ao mesmo tempo em que a população com mais de 65 anos vai continuar aumentando nas próximas décadas. Esses dados indicam que estamos vivenciando um processo acelerado de transição demográfica, o que traz muitos desafios para os gestores públicos que precisam desenvolver políticas direcionadas a esta população com idade cada vez mais avançada. Ao mesmo tempo, a população considerada ativa (14-65 anos) vai se tornando menor do que a população considerada inativa (0-14 e mais de 65 anos), trazendo sérias consequências para a manutenção do sistema previdenciário, uma vez que o número de aposentados passa a ser superior ao número de contribuintes. (FEE, 2014). Outro fator que necessita ser melhor investigado é o do retorno ou da permanência dos idosos no mercado de trabalho, provocando um envelhecimento da população ativa. Este aspecto, que já pode ser verificado empiricamente, como iremos demonstrar mais adiante, ainda precisa passar por análises mais minuciosas para desvendar as razões que podem estar associadas a este fenômeno, o qual pode tanto representar a busca de uma vida ativa por parte dos idosos, como a necessidade de 
AREOSA, S. V. C.; FREITAS, C. R.; LAMPERT M.; TIRELLI, C.

uma complementação salarial devido à precarização das suas condições de vida e da sua família.

Além das questões ligadas à idade, também se observa uma diferença em relação ao gênero, pois os dados indicam que vem ocorrendo uma feminilização crescente da velhice, posto que a expectativa de vida das mulheres idosas é significativamente superior à dos homens. (BATISTA; ALMEIDA; LANCMAN, 2011).

Essas mudanças vêm atingindo distintas áreas das políticas públicas, pois trazem novas demandas para as áreas da assistência, da saúde, da educação, trabalho, mobilidade urbana, entre outras. Diante dessa nova configuração populacional, o Governo Federal Brasileiro vem, desde a década de 1990 instituindo políticas direcionadas aos idosos, como a Política Nacional do Idoso -PNI (BRASIL, 1994), a qual forneceu as principais diretrizes das ações destinadas aos idosos e previu a criação de conselhos em todas as unidades da federação. Nos anos subsequentes foram criadas a Política de Saúde do Idoso, em 1999, o Estatuto do Idoso (EI), em 2003, e a Política Nacional de Saúde da Pessoa Idosa, em 2006. Segundo Batista, Almeida e Lancman (2011, p. 203), esta última incorporou os elementos previstos na Política do Envelhecimento Ativo da Organização Mundial da Saúde (OMS, 2005), na qual aparece, no âmbito da participação, "[...]o incentivo à educação e oportunidades de aprendizagem durante o curso de vida, envolvimento ativo dos idoso nas atividades de trabalho formal, informal e voluntário e sua participação, de forma integral, na vida familiar e comunitária."

No contexto de um envelhecimento ativo, nota-se que a população idosa tem buscado atividades voltadas à educação e ao conhecimento como um processo contínuo. Diante desse cenário, as universidades, a partir da década de 90, têm oferecido serviços específicos para idosos, como as Universidades Abertas para a Terceira Idade (UNATI's). Essas universidades objetivam oferecer ações de ensino, pesquisa e extensão voltadas para pessoas em processo de envelhecimento, almejando reinserir o idoso socialmente a partir da criação de novas oportunidades de estudo, as quais buscam resgatar e reafirmar a sua independência e autonomia.

Contudo, além dos programas voltados para a Terceira Idade e as UNATI's, tem-se verificado nas universidades um novo fenômeno entre a população idosa. Esse fato refere-se à busca de um curso de graduação, em nível superior, por idosos com 60 anos ou mais. A inserção do idoso em cursos de graduação difere da entrada nas ações propostas pela Universidade que são específicas para a população idosa. No processo de ingresso para a graduação, a pessoa idosa realiza o concurso vestibular, concorrendo com jovens de todas as 
ENVELHECIMENTO ATIVO: UM PANORAMA DO INGRESSO DE IDOSOS NA UNIVERSIDADE

idades e, em caso de aprovação, passa a integrar as turmas de graduação com esses mesmos jovens.

A Universidade de Santa Cruz do Sul (UNISC) oferece, atualmente, aproximadamente 50 cursos de graduação presenciais e três (03) cursos à distância. Dentre esses cursos, encontram-se estudantes de todas as idades, o que proporciona um espaço intergeracional em sala de aula, onde se relacionam idosos com 60 anos ou mais e alunos recém-saídos do Ensino Médio. As ações para o envelhecimento com qualidade de vida nesta universidade vêm sendo desenvolvidas desde 1993, o que demonstra o comprometimento da UNISC com a garantia de direitos da população idosa residente nos municípios do Vale do Rio Pardo, reafirmando seu compromisso comunitário e sua preocupação com o desenvolvimento de toda população.

A entrada crescente desses idosos em diferentes cursos de graduação coloca-nos diante de algumas questões: Por que os idosos têm buscado cursar graduações junto com os segmentos mais jovens da população e não apenas nas atividades e cursos direcionados à sua faixa etária? Esta busca pode ser interpretada como a procura de uma formação para se inserir no mercado de trabalho? Essas questões serão discutidas, a seguir, com base na literatura e nos dados empíricos coletados.

Este artigo encontra-se estruturado em duas seções, além desta introdução e dos comentários finais. Na primeira seção, aborda-se a questão do envelhecimento ativo, a contribuição da Gerontologia Social e o surgimento das Universidades Abertas da Terceira Idade (UNATI's); na segunda seção, apresenta-se os dados empíricos referentes a inserção dos idosos nos cursos de graduação da UNISC e discute-se as suas possíveis interpretações, sobretudo em relação às modificações observadas no mercado de trabalho. Por fim, são apresentadas as considerações finais.

\section{ENVELHECIMENTO, GERONTOLOGIA E AS UNATI'S}

Ao se pensar no envelhecimento e suas relações com a saúde e o contexto social, verifica-se que ocorrem alterações estruturais e funcionais nos indivíduos idosos. Essas mudanças são parte de um processo natural de evolução da idade e podem se manifestar de formas diferentes, de acordo com as características individuais e socioeconômicas. Neste sentido, o modo como um indivíduo envelhece não depende somente de fatores genéticobiológicos, mas também do contexto social cujos fatores associados comumente estão fora de 
AREOSA, S. V. C.; FREITAS, C. R.; LAMPERT M.; TIRELLI, C.

controle, como por exemplo, a presença da pobreza, a dificuldade de acesso à educação ou a serviços de promoção da saúde e prevenção de doenças. (FIGUEIREDO, 2008). Hoje, tem-se verificado que a genética seria responsável por cerca de $25 \%$ da variação da longevidade e que os fatores ambientais seriam responsáveis por cerca de 50\%, o que corrobora a importância dos aspectos sociais do indivíduo na forma como o envelhecimento vai se apresentar. (HJELMBORG et al., 2008).

Pode-se classificar o envelhecimento como bem-sucedido ou usual. Entende-se por bem-sucedido quando o indivíduo apresenta diminuições fisiológicas com o passar dos anos sem que essas alterações, determinadas pela idade sofram complicações devido a doenças, exposições ambientais desfavoráveis ou fatores associados a estilo de vida. Neste caso, observa-se uma capacidade fisiológica maior e um risco menor de adoecimento, o que demonstra a existência de componentes previsíveis ou reversíveis, que antes eram considerados como parte do envelhecimento normal. Já o envelhecimento usual, corresponde à alterações determinadas por efeitos combinados do processo de envelhecimento e pelos efeitos de doenças e de fatores adversos do ambiente e do estilo de vida. (BRITTON et al., 2008).

Ao se pensar em envelhecimento satisfatório ou bem-sucedido, percebe-se que a velhice parece estar mais sob controle do que se acreditava previamente. O número de alterações relacionadas com a idade, pensadas anteriormente como inevitáveis e trazendo diversas perdas associadas são, provavelmente, em número muito menor do que se esperava. Sendo assim, bastaria evitar, ao que parece, que o efeito do envelhecimento fosse agravado pelo estilo de vida adotado ou por fatores ambientais, e se teria um envelhecimento bem-sucedido no lugar do usual. De acordo com Ferrari (2002) um envelhecimento saudável ou bemsucedido reside na possibilidade de suscitar atitudes ativas durante a utilização do tempo livre, como a participação consciente e voluntária na vida social, opondo-se ao isolamento e ao recolhimento social. Visando dar conta das questões trazidas com o processo de envelhecimento crescente da população desenvolveu-se, na segunda metade do séc. XX, uma área de estudos específica, a Gerontologia, a qual será descrita a seguir.

Com o aumento da população idosa a nível mundial, iniciaram-se as reflexões e movimentos por parte da sociedade e de seus governantes em relação à temática. (MAZO et al., 2004). Para dar conta desse fenômeno, surgiu o conceito de Gerontologia como a ciência que estuda o envelhecimento. Esta ciência obteve um desenvolvimento crescente após a II Guerra Mundial com o objetivo de contribuir para uma maior longevidade com qualidade de 
ENVELHECIMENTO ATIVO: UM PANORAMA DO INGRESSO DE IDOSOS NA UNIVERSIDADE

vida no período denominado de "velhice". Desse modo, a Gerontologia tem como objetivo tratar dos aspectos biológicos, sociais, psíquicos, legais, entre outros e promover pesquisas que possam estabelecer os fatores envolvidos em sua gênese. Segundo Papaléo Netto (2006), o envelhecimento apresenta desafios ligados à sua natureza e dinâmica, mesmo sendo um fenômeno comum a todos os seres vivos.

A Gerontologia tornou-se hoje, um campo multidisciplinar e multiprofissional dedicado ao estudo da velhice, designada como a última fase do curso vital, onde o protagonista é considerado o "idoso", aquela pessoa com 60 anos ou mais, assim classificada em função de suas características num determinado contexto histórico e social. Esta ciência vem agregando, cada vez mais, profissionais e pesquisadores de todas as áreas do conhecimento, os quais buscam dentro da sua especificidade contribuir para o estudo do envelhecimento. Os profissionais que trabalham com o envelhecimento humano sabem que este é um fenômeno multifacetado e multifatorial.

A busca por conhecimento científico e específico do processo de envelhecimento e sua aproximação com a comunidade fez com que as primeiras ações e atividades voltadas para os idosos começassem a surgir nos anos de 1960. A França criou as Universidades do Tempo Livre, que foram precursoras das Universidades da Terceira Idade surgidas em 1973, pelas mãos de Pierre Vellas, um reconhecido professor de Direito Internacional da Universidade de Ciências Sociais de Toulouse. Desde o início, os objetivos das Universidades Abertas para a Terceira Idade foram retirar os idosos do isolamento, propiciar-lhes saúde, energia e interesse pela vida, além de modificar sua imagem perante a sociedade. (CACHIONI, 2003). As UNATI's que surgiram na França na década de 1970 foram reformuladas, sendo que as Instituições de Ensino Superior distribuídas em vários países criaram o seu modelo para trabalhar com esta população. Em termos gerais, as ações ofertadas pelas universidades são voltadas à valorização da vida do idoso, reinserindo-o socialmente, criando novas oportunidades de estudo, resgatando e reafirmando sua independência e autonomia.

No início da década de 1980, este tipo de programa chegou à América Latina através das Universidades Abertas - UNI3 Uruguai, com sede no Instituto de Estudos Superiores de Montevidéu - com base nos princípios da UNI3 de Genebra. O Uruguai foi o primeiro país da América do Sul a implantar o modelo de Universidade Aberta para a Terceira Idade a qual se estendeu por outros países como o Paraguai, a Argentina, o Chile, o Panamá, a Venezuela, o México e o Brasil. No Brasil, especificamente, a Universidade para a Terceira Idade se instalou em Passo Fundo, no estado do Rio Grande do Sul. (CACHIONI, 2003). Outras 
AREOSA, S. V. C.; FREITAS, C. R.; LAMPERT M.; TIRELLI, C.

Instituições de Ensino Superior (IES) também foram criando as suas ações de forma isolada, através de atividades de extensão, ou de forma integrada, como as UNATI's. Segundo relatos dos próprios idosos no artigo "A influência da Universidade Estadual do Sudoeste da Bahia (UESB) no processo de viver e envelhecer dos idosos estudantes/integrantes", estar dentro da universidade "abriu a cabeça da gente", "hoje é que estou me sentindo jovem”, "entendi que estou viva", "na UATI (Universidade Aberta à Terceira Idade) passei a ter outra conscientização porque antes ao invés de se valorizar, a gente mesmo se desvalorizava”. Com isso, "constatamos que a UATI/UESB contribuiu significativamente para a melhoria da qualidade de vida, com apoio ao exercício da cidadania, a qual envolve diversos aspectos do viver e envelhecer". (SENA et al., 2003, p.39). A partir disso, as universidades em todo o país têm aderido à proposta positivamente, criando programas que visam atender essa parcela da população. Percebe-se, por meio dessas falas, que a convivência no ambiente acadêmico "quebra" o estigma de que o idoso não produz mais, não tem mais capacidade de aprendizagem ou que não pode realizar atividades que normalmente são feitas por pessoas mais jovens, como estudar e aprender coisas novas.

Um dos grandes desafios para quem trabalha com educação é integrar o ensino de estratégias de aprendizagem ao longo dos diversos anos de escolaridade e, entre as várias gerações que se encontram em uma sala de aula de um curso de graduação. É preciso contribuir para que o acadêmico possa desenvolver os processos de auto regulação, incentivar a autonomia e o controle que o estudante pode desempenhar na aprendizagem, atendendo aos diferentes contextos. (SIMÃO; FRISON, 2013).

Os programas de terceira idade das universidades, além da oportunidade da reinserção nos estudos, ofertam também oficinas, grupos de convivência, entre outras atividades. As oficinas trabalham áreas como a memória, a criatividade, o contato com as novas tecnologias e a saúde física, promovendo atividades como ginástica, hidroginástica e dança. Esses últimos possuem importância fundamental não só pela interação social, mas por promover a saúde, estimular o cuidado com o corpo e aumentar a socialização dos idosos. Com essas ações, a universidade possibilita a participação e integração dos idosos no meio acadêmico respondendo a sua responsabilidade neste século XXI, socializando saberes e produzindo novos conhecimentos e práticas interdisciplinares inovadoras. (DEL-MASSO, 2009). Para Ferrari (2002, p. 103), as atividades das Universidades para o envelhecimento têm contribuído para: i) facilitar a oportunidade grupal, de sociabilização, de participação, de vivência de manutenção dos direitos e papéis sociais; ii) ajudar o idoso através das diferentes atividades a 
ENVELHECIMENTO ATIVO: UM PANORAMA DO INGRESSO DE IDOSOS NA UNIVERSIDADE

vencer sua constante incapacidade para lidar com perdas múltiplas; iii) manter e adaptar pelo maior tempo possível a sua independência física, mental e social; iv) estimular o indivíduo para realizar atividades visando o treinamento sensorial e o desenvolvimento da criatividade; v) reconstruir padrões de vida e atividades; e vi) avaliar o desempenho adaptativo do idoso como um dos indicadores de saúde.

Tem-se observado, nas últimas décadas, um aumento de estudos teóricos e empíricos que buscam compreender os processos psicológicos que fazem com que os indivíduos dirijam seu comportamento para alcançar metas pessoais de forma a fazer opções que melhor se adequem à concretização de seus anseios. (SIMON; FRISON, 2013). Neste sentido, as pessoas de mais idade também estão interessadas em atividades que possuam um significado pessoal para elas. E, levando em conta que a participação do idoso só ocorrerá se o foco for de seu interesse e motivação, torna-se imprescindível que as Universidades possam alcançar os objetivos dos idosos nos programas das UNATI's. (ZAWADSKI; VAGETTI, 2007).

\section{A INSERÇÃO DE IDOSOS NA UNISC: A BUSCA POR UM ENVELHECIMENTO ATIVO}

Atenta ao movimento mundial e nacional e almejando avançar rumo à criação da Universidade Aberta à População Idosa, a Universidade de Santa Cruz do Sul (UNISC), em 1998, propôs a criação do Programa Terceira idade na UNISC, integrando diversas áreas na extensão e propondo ações de pesquisa interdepartamental. Atualmente, são treze (13) os projetos de extensão que atendem idosos na Universidade, com ações em Saúde, Informática e Comunicação Social. Mais de dois mil idosos do município de Santa Cruz do Sul e da região são atendidos nos projetos e ações da UNISC. A UNISC, como Universidade Comunitária comprometida com o desenvolvimento regional, busca promover ações institucionais definidas, prioritariamente, a partir de demandas e necessidades da sociedade.

A abertura da universidade para a população idosa foi uma das demandas sociais contempladas nos últimos anos. A primeira turma da Universidade Aberta para o Adulto Maior (UNIAMA), criada em 2014, recebeu 30 inscrições e foi composta por 28 idosos, sendo 23 do sexo feminino e cinco do sexo masculino. Mesmo sendo um projeto recente, a UNIAMA possui grande demanda por parte dos idosos. Na interação com esses estudantes, observa-se a acentuada procura e interesse pela educação continuada e, principalmente, pela socialização que a universidade propicia. 
AREOSA, S. V. C.; FREITAS, C. R.; LAMPERT M.; TIRELLI, C.

Porém, a UNIAMA não é o único espaço que os idosos vêm ocupando na UNISC. Cada vez mais se percebe a inclusão de pessoas de mais idade nos bancos escolares e nas turmas regulares de graduação. Este fato torna-se um desafio para os estudos sobre o envelhecimento, à medida que pode indicar a busca por uma nova inserção profissional por parte de idosos, bem como a vontade de interagir com pessoas de diferentes faixas etárias. De qualquer forma, a inserção crescente dos idosos nos cursos de graduação da UNISC aponta para a perspectiva de um envelhecimento bem-sucedido ou ativo.

Para demonstrar a inserção dos idosos nos cursos de graduação da UNISC, buscou-se levantar dados comparativos sobre o número de pessoas com mais de 60 anos matriculadas em cursos regulares. A seguir, serão apresentados os dados quantitativos referentes aos idosos inscritos em cursos de graduação, para os anos de 2004 e 2015. Este levantamento foi realizado junto aos vários setores que fazem atendimento à idosos na UNISC, no segundo semestre de 2004 e de 2015. Os resultados de 2004 fazem parte do banco de dados do grupo de pesquisa "Realidade, Exclusão e Cidadania na Terceira Idade" e foram acrescidos pelos resultados encontrados em 2015 para fins comparativos. Os resultados mostram um crescimento no uso dos espaços abertos para essa população na universidade, já tendo sido contabilizados mais de 2000 idosos participantes dos vários serviços oferecidos pela instituição. Inicialmente, esse incremento se deu através da abertura de atividades de extensão e, posteriormente, por meio de incentivo e desconto na graduação, o que se refletiu nas mudanças descritas na Tab. 1, exposta a seguir.

Tabela 1: Estudantes com mais de 60 anos por curso de graduação

\begin{tabular}{c|c|c|c|c|c}
\hline \multicolumn{2}{c}{2004} & \multicolumn{3}{c}{2015} \\
\hline CURSO & MULHERES & HOMENS & CURSO & MULHERES & HOMENS \\
\hline Psicologia & 0 & 1 & Psicologia & 4 & 2 \\
\hline Direito & 0 & 1 & Direito & 4 & 4 \\
\hline $\begin{array}{c}\text { Pedagogia - } \\
\text { Licenciatura }\end{array}$ & 1 & 0 & $\begin{array}{c}\text { Pedagogia - } \\
\text { Licenciatura }\end{array}$ & 1 & 0 \\
\hline $\begin{array}{c}\text { Gestão e } \\
\text { Direito } \\
\text { Tributário }\end{array}$ & 0 & 2 & Serviço Social & 1 & \\
\hline & & & Gastronomia & 2 & 1 \\
\hline & & & Turismo & 1 & 0 \\
\hline & & & $\begin{array}{c}\text { Arquitetura e } \\
\text { Urbanismo }\end{array}$ & 1 & 0 \\
\hline & & & Engenharia & 0 & 1 \\
\hline
\end{tabular}


ENVELHECIMENTO ATIVO: UM PANORAMA DO INGRESSO DE IDOSOS NA UNIVERSIDADE

\begin{tabular}{l|l|l|l|l|c}
\hline & & & Civil & & \\
\hline & & & Filosofia & $\mathbf{1}$ & $\mathbf{0}$ \\
\hline & & & Letras & $\mathbf{1}$ & $\mathbf{0}$ \\
\hline
\end{tabular}

Fonte: Informações elaboradas pelas autoras a partir dos dados da Secretaria acadêmica da UNISC

Conforme a Tab. 1, nos últimos dez anos pode-se perceber um aumento de $400 \%$ no ingresso de acadêmicos, com mais de 60 anos, em cursos de graduação na UNISC. Em 2004, havia cinco alunos matriculados em quatro cursos e hoje existem 25 alunos inseridos em onze cursos de graduação. As formações procuradas em 2004 pela população idosa continuam a ser buscadas em 2015, porém, os idosos que hoje estão na graduação diversificaram as suas escolhas e almejam cursos profissionalizantes como a gastronomia e cursos das áreas das ciências exatas e sociais aplicadas, como as engenharias, arquitetura, administração, entre outras. Esse fato pode demonstrar o desejo de obter uma nova formação com intenções de retornar ao mercado de trabalho, a partir de uma outra profissão.

De acordo com Vieira (2010), o ingresso na universidade para pessoas na Terceira Idade é um privilégio oferecido pela sociedade contemporânea, possibilitando a ampliação de conhecimentos em diversos cursos. Essas atividades também são uma oportunidade de ampliação do círculo de amizades e da possibilidade de surgimento de laços amorosos entre os idosos dentro da universidade, o que vem aumentando gradativamente. Conforme Simon e Frison (2013) uma prática dialógica em situações de interação e troca, possibilita a ativação e a manutenção das ideias, motivações, comportamentos e afetos dos alunos idosos. Da mesma forma, os processos de ensino e aprendizagem implicam, necessariamente, a presença de interações sociais, o que acaba tendo maior ênfase entre a população mais velha na medida em que est está mais sujeita ao isolamento. Esses aspectos também são relevantes no sentido de ampliar as probabilidades de um envelhecimento bem-sucedido, diante da variedade de formas pelas quais se constroem a velhice.

Em relação à categoria gênero, também se verificaram mudanças ao longo dos últimos anos nos alunos idosos dos cursos de graduação da UNISC. Enquanto em 2004 havia apenas uma mulher frequentando aulas na graduação, em 2015, existiam 17 alunas matriculadas. Hoje, as mulheres idosas são a maioria nos cursos de graduação, o que demonstra que elas conseguem acessar, cada vez mais, os bancos escolares, o que pode lhes trazer novas possibilidades de inserção no mercado de trabalho. Outra questão presumível para o aumento das mulheres idosas em cursos de graduação pode-se dar pelo fato de que, com a diminuição 
AREOSA, S. V. C.; FREITAS, C. R.; LAMPERT M.; TIRELLI, C.

das tarefas relacionadas à família e a casa, a partir da chegada da velhice, a mulher pode realizar atividades que anteriormente não conseguia como, por exemplo, frequentar um curso de graduação. Além desses fatores, o fato das mulheres constituírem a maioria da população idosa também deve pesar na sua maior representatividade dentre os alunos que frequentam os curso de graduação.

Conforme Capelle et al. (2007, p. 503), a inserção da população feminina nas organizações tem alterado o comportamento da mão-de-obra e das relações de trabalho na sociedade contemporânea. Esses autores afirmam que as mulheres estão conquistando maiores espaços "em praticamente todas as atividades, mas, apesar dessa conquista e de possuírem os mesmos ou melhores níveis de escolaridade que os homens, ainda existem algumas disparidades quando se discute a igualdade entre gêneros". Além disso, também destacam que o nível de escolaridade das mulheres tem sido mais elevado do que o dos homens que ocupam as mesmas funções/cargos que elas. Desse modo, o fator gênero tem se constituído como um dos elementos fundamentais para se pensar o processo de envelhecimento, mostrando que há diferenças na forma de envelhecer entre homens e mulheres.

A compreensão da velhice como uma categoria heterogênea e sua articulação com outras dimensões da vida social tem permitido muitos recortes analíticos que vem se mostrando bastante frutíferos para a compreensão dos múltiplos aspectos relacionados às formas de envelhecimento em cada sociedade. A preparação para a velhice, as condições econômicas e sociais e também as diferenças de gênero são fatores importantes a serem observados na maneira como se desenvolve o envelhecer e que se diversificam entre mulheres e homens em idade avançada.

Outro aspecto interessante a ser observado no perfil dos alunos idosos dos cursos de graduação da UNISC são as mudanças em relação à idade desses alunos. A turma que frequentava os cursos em 2004, possuía idades entre 61 e 66 anos. Hoje, tem-se um perfil de idosos com idades mais avançadas, entre 60 e 70 anos. Isso reforça a ideia do aumento da longevidade, demonstrando que as pessoas estão bem fisicamente e cognitivamente, o que reforça o desejo pela realização profissional nesta fase da vida. A profissionalização passa a não ser apenas um sonho de jovens, mas também de velhos.

Algumas pesquisas voltadas à identificação dos preditores de boa capacidade funcional, nos domínios físico e cognitivo durante o processo de envelhecimento, têm indicado correlações com fatores de ordem socioeconômica e de estilo de vida. Em um estudo 
ENVELHECIMENTO ATIVO: UM PANORAMA DO INGRESSO DE IDOSOS NA UNIVERSIDADE

longitudinal desenvolvido por Perls et al.(2002), o envelhecimento bem-sucedido foi identificado em $12,8 \%$ dos homens e $14,6 \%$ das mulheres. Como preditor mais forte de envelhecimento bem sucedido foi encontrado a posição socioeconômica do idoso. E, após o ajuste desse fator, observou-se relação com a adoção de hábitos saudáveis para as mulheres e a presença de um trabalho para os homens, demonstrando a existência de fatores sociais associados a melhores resultados na forma de envelhecer, além de subsidiar a compreensão acerca do crescente interesse dos idosos pela inserção em cursos de graduação e sua manutenção no mercado de trabalho.

A permanência ou reinserção da população idosa no mercado de trabalho pode ser considerada uma das transformações sociais importantes trazidas pelo novo milênio. O IBGE, já em 2002, demonstrava o aumento de trabalhadores com mais de 40 anos. Buaes (2004) afirma que o mercado de trabalho absorverá, no futuro, um número reduzido de jovens e um número cada vez maior de trabalhadores com mais idade. O crescimento do número de trabalhadores mais velhos já tem sido observado na população economicamente ativa (PEA). Segundo os dados do IBGE, em 2006 os trabalhadores com mais de 50 anos representavam $18,1 \%$ da população total de trabalhadores das seis regiões metropolitanas.

No censo de 2010 observa-se que o percentual de pessoas com mais de 60 anos que permanecem no mercado de trabalho é de 3,34\% no Brasil, 4,59\%, no Rio Grande do Sul e $5,66 \%$, no Vale do Rio Pardo (VRP). No VRP, a população economicamente ativa (PEA) soma 240.202 pessoas, $65,57 \%$ da população total; destes, $20.741(5,66 \%)$ são idosos. Os homens idosos constituem maioria dentre a população economicamente ativa, perfazendo $11.904(3,24 \%)$ homens frente à $8.839(2,41 \%)$ mulheres idosas. No VRP há um número maior de idosos economicamente ativos que residem na zona rural, os quais somam 12.485 $(3,4 \%)$ idosos, em comparação aos 8.248 (2,24\%) que residem no meio urbano. Essa predominância de idosos economicamente ativos na área rural explica-se pela maior densidade demográfica de idosos nesse espaço. Este cenário aponta para a necessidade de investimentos em estratégias tanto na manutenção do desenvolvimento da força de trabalho madura quanto na busca de melhores condições para o envelhecimento no trabalho. (IBGE, 2010).

A mudança acelerada no padrão demográfico no Brasil tem acarretado novas demandas para as políticas públicas, sobretudo às relacionadas aos serviços de saúde e de seguridade social. As modificações que vêm ocorrendo na taxa de participação dos idosos no meio econômico alteram a oferta de trabalho total, impactando nos níveis de ocupação de todos os 
AREOSA, S. V. C.; FREITAS, C. R.; LAMPERT M.; TIRELLI, C.

outros grupos de idade. Percebe-se que, a relação entre trabalhadores economicamente ativos e inativos vem diminuindo, ao passo que a expectativa de sobrevida em idades mais avançadas está aumentando. (FURTADO, 2005).

Este aumento da proporção de pessoas mais velhas na população economicamente ativa demonstra que a população brasileira está passando por mudanças significativas que colocam novos desafios aos formuladores de políticas públicas e à sociedade como um todo. Por um lado, as mudanças recentes trazidas por um maior envelhecimento populacional e redução da taxa de fecundidade podem ser vistas como uma oportunidade única de enriquecimento e desenvolvimento do país, associada ao acúmulo de riqueza advindo do aumento da proporção de pessoas em idade ativa em relação aos grupos etários dependentes. Por outro lado, para que ocorra um bom aproveitamento dessas transformações, faz-se necessário a criação de melhores empregos, a redução das desigualdades socioeconômicas e a reestruturação das políticas de gestão de pessoas, no âmbito empresarial e na ação sindical. (DIEESE, 2013).

Para se efetivar várias dessas medidas, o Estado precisa atuar no sentido de promover a implantação de políticas públicas que harmonizem os territórios fragmentados em prol dos interesses públicos, tendo em vista que as desigualdades no processo de desenvolvimento se devem, basicamente, às condições desiguais de vida e de trabalho a que estiveram submetidas às pessoas que, atualmente, são idosas. (PAMPLONA, 2012).

A partir da análise dos dados coletados e da literatura sobre envelhecimento, percebe-se que uma parcela da população idosa tem buscado alcançar uma melhor qualidade de vida e um envelhecimento bem sucedido através da realização de atividades físicas e cognitivas. Dentre essas atividades, destacamos aquelas oferecidas pelas universidades através de cursos de extensão, criação de UNATIs ou da inserção dos idosos em cursos regulares da Graduação. Em nosso entender, a qualidade de vida está vinculada tanto com o bem-estar subjetivo, quanto com os elementos objetivos, como bens materiais e serviços, os quais são indispensáveis para a manutenção da dignidade humana. O fato dos idosos estarem procurando, de forma crescente, se inserirem em cursos regulares de graduação pode estar associado tanto à vontade de socializar com pessoas mais jovens, abrindo possibilidades de renovação de relações e de valores, como pode representar a busca por uma nova possibilidade de profissionalização e inserção no mercado de trabalho, trazendo uma maior realização pessoal e/ou material. Para que se possa compreender as motivações que levam esses idosos aos bancos universitários necessitamos empreender mais investigações de caráter qualitativo. Por ora, sabemos que existe um interesse crescente, por parte da população idosa, 
ENVELHECIMENTO ATIVO: UM PANORAMA DO INGRESSO DE IDOSOS NA UNIVERSIDADE

em prosseguir os estudos e realizar atividades em conjunto com pessoas de diferentes faixas etárias. Diante disso, as universidades vêm respondendo a essas novas demandas por meio da realização de vários tipos de ação que visam facilitar e viabilizar a entrada de idosos no espaço acadêmico.

\section{CONSIDERAÇÕES FINAIS}

Quando se fala em educação permanente acredita-se que esta deve perpassar todos os ciclos vitais para que corresponda às necessidades da sociedade moderna. Assim, vê-se que a estruturação da educação não pode se limitar a um período particular da vida, mas deve permitir ao indivíduo que aprenda ao longo de todo o seu curso de vida. Portanto, vê-se no aumento da inserção de idosos em cursos de graduação da UNISC uma crescente atuação desta universidade quanto à disponibilização de espaços para uma educação continuada.

Uma das preocupações constantes na vida daquele que envelhece é a perda da saúde e da sua autonomia e independência. Uma forma de garantir o seu bem-estar passa pela realização de diversos tipos de atividades, a convivência em grupos e a concepção de novos projetos de vida, como o representado por uma nova graduação, que pode auxiliar na manutenção da sua autonomia e saúde física e psicológica. As relações sociais que os idosos estabelecem nos grupos são um importante aliado para preencher o vazio que alguns idosos sentem em sua vida devido às perdas que acompanham esta faixa etária e servem para preservar a sua qualidade de vida.

Além disso, presenciamos, cada vez mais, o aumento do número de pessoas com mais de 60 anos que ainda se encontram no mercado de trabalho. E, desta forma, a busca por uma graduação, nesta faixa etária, pode também representar o desejo de vir a se inserir no mercado de trabalho, mesmo estando em idade avançada, ou a busca por uma outra profissão. Seriam importantes, nesse sentido, a realização de pesquisas qualitativas que possibilitassem desvendar os motivos associados a este fenômeno.

\section{ACTIVE AGEING: AN OVERVIEW OF THE UNIVERSITY ENTRANCE OF}

\section{ELDERLY}


AREOSA, S. V. C.; FREITAS, C. R.; LAMPERT M.,; TIRELLI, C.

\begin{abstract}
The paper presented here builds on the social context present nowadays whose highlight is the population aging and longevity as challenges to society. the main objective is to present data on the inclusion of seniors in undergraduate courses in the Universidade de Santa Cruz do Sul/RS. The increase of the elderly in higher education shows a new facet of aging, marking the possibility of a successful aging with quality of life. Thus, presents comparative data the inclusion of seniors in undergraduate courses in 2004 and 2015. The increasing proportion of older people of working age, seeking a new training, may represent beyond the occupation of free time, insertion into the labor market and a perspective for this stage of life.
\end{abstract}

Keywords: Active Ageing, University, Social Development

\title{
ENVEJECIMIENTO ACTIVO: UNA VISIÓN GENERAL DE LA ENTRADA DE LAS PERSONAS MAYORES EN LA UNIVERSIDAD
}

\section{Resumen}

Este estudio se basa en el contexto social destacando este envejecimiento de la población y la longevidad como desafíos para la sociedad de hoy. Por lo tanto, su principal objetivo es dar a conocer datos sobre la inclusión de las personas mayores en los cursos de graduación en la Universidad de Santa Cruz do Sul / RS. El aumento de las personas mayores en la educación superior muestra una nueva faceta de envejecimiento, que marca la posibilidad de un envejecimiento exitoso con calidad de vida. Por lo tanto, se presentan datos comparativos inserción de las personas mayores en los cursos de graduación en 2004 y 2015. El aumento de la proporción de personas mayores en edad de trabajar que la búsqueda de una nueva formación, puedan representar más allá de la ocupación del tiempo libre, integración en el mercado laboral y una nueva perspectiva para esta fase de vida. 
ENVELHECIMENTO ATIVO: UM PANORAMA DO INGRESSO DE IDOSOS NA UNIVERSIDADE

Palabras-clave: Envejecimiento Activo; Universidad; Desarollo Social

\section{REFERÊNCIAS}

ARAÚJO, A. O; GUARATO, D. Z. Envelhecimento e Saúde Mental. In: COSTA, G. A. Atividade física, envelhecimento e a manutenção da saúde. Uberlândia: Edufu, 2010.p.188

BATISTA, M. P. P., et a. Políticas públicas para a população. Revista de Terapia Ocupacional da Universidade de São Paulo, São Paulo: Universidade de São Paulo, vol.22, n.3, p.200-207, set./dez. 2011.

BRASIL. Ministério da Educação. A democratização e expansão da educação superior no país 2003 - 2014. 2015. Disponível em: <http://portal.mec.gov.br/index.php?Option =com_content\&view=article\&id=20954\&Itemid=1329>. Acesso em: maio 2015.

BRITTON, A. et al. Successful aging: the contribution of early-life and midlife risk factors. Journal of the American Geriatricts Society, vol.56, n.6, p. 1098-1105, 2008.

BUAES, Caroline Stumpf. Envelhecimento e educação: em foco a aprendizagem de trabalhadores mais velhos. Estudos interdisciplinares sobre o envelhecimento, vol. 6, n.1, p.720, 2004.

CACHIONI, Meire. Quem educa os idosos: Um estudo sobre professores de Universidades da Terceira Idade. Campinas, SP: Editora Alínea, 2003.

DEL-MASSO, Maria Candida Soares. Envelhecimento Humano e Qualidade de Vida: Responsabilidade da Universidade neste século XXI. UNICAMP. Campinas, 2009. Disponível em:

<http://www.fef.unicamp.br/fef/qvaf/livros/foruns_interdisciplinares_saude/evolucao/evoluca o_cap3.pdf>. Acesso em: 15 maio 2013.

DIEESE - DEPARTAMENTO INTERSINDICAL DE ESTATÍSTICA E ESTUDOS SOCIOECONOMICOS. Nota Técnica 127. Os desafios à ação sindical decorrentes das mudanças na população (o que mudou, o que está mudando, o que vai mudar e o que ainda não mudou). p 1-19, junho/2013. Disponível em:

<http://www.dieese.org.br/notatecnica/2013/notaTec127demografia.pdf > . Acesso em: 25 set. 2014.

FEE. Dinâmicas territoriais recentes no Estado do Rio Grande do Sul /coordenação de Antonio Paulo Cargnin. - Porto Alegre: FEE, 2014. - (RS 2030: agenda de desenvolvimento territorial) v. : il. 
AREOSA, S. V. C.; FREITAS, C. R.; LAMPERT M.,; TIRELLI, C.

FIGUEIREDO, M. L. F., et al. Diagnóstico de enfermagem do idoso acamado no domicilio. Revista Brasileira de Enfermagem, Brasília: Associação Brasileira de Enfermagem, vol. 61, n.4, p. 464-9, 2008.

FURTADO, Adolfo. A participação do idoso no mercado de trabalho brasileiro. Biblioteca Digital da Câmara dos Deputados: Brasília-DF, p.1-26, 2005. Disponível em:

<http//:bd.camara.gov.br>. Acesso em: 27 set. 2014.

HJELMBORG, V. B. J.; IACHINE, I.; SKYTTHE, A. Genetic influence on human lifespan and longevity. Journal of Human Genetics, vol. 119, n.3, p-312-321, 2006.

IBGE - Instituto Brasileiro de Estatística e Geografia. Censo Demográfico 2010. Informações completas. Disponível em: 〈http://www.sidra.ibge.gov.br/cd/cd2010RgaAdAgsn.asp〉. Acesso em: 23 ago. 2014.

MAZO, G. Z.; LOPES, M. A; BENEDETTI, T. B. Atividade física e o idoso: concepção gerontológica. Porto Alegre: Sulina, 2004.

NERI, Anitta Liberalesso. Teorias Psicológicas do envelhecimento: percurso histórico e teorias atuais. In: FREITAS, E. V. et al. Tratado de Geriatria e Gerontologia. 2. ed. Rio de Janeiro: Guanabara Koogan, 2006.

PALMA, L. S.; CACHIONI, M. Educação Permanente: perspectiva para o trabalho educacional com o adulto maduro e com o idoso. In: FREITAS, E. V. (Orgs.). et al. Tratado de Geriatria e Gerontologia, Rio de Janeiro: Guanabara Koogan, 2002, p. 1101-1121.

PAMPLONA, Leonardo de Moura Perdigão. Políticas públicas territoriais: uma nova visão sobre o desenvolvimento. In: Premio Celso Furtado de Desenvolvimento Regional, 2012. Relatório Técnico. Brasília: Ministério da Integração Nacional, 2012, p. 75-81.

PAPALÉO NETTO, Matheus. Gerontologia: a velhice e o envelhecimento em visão globalizada. São Paulo: Atheneu, 2002.

PERLS, T. T; et al. Life-long sustained mortality advantage of siblings of centenarians. Proceedings of the National Academy of Sciences, vol. 99, n.12, p. 8442-8447, 2002.

SENA, Edite Lago da Silva et al. A influência da Universidade Estadual do Sudoeste da Bahia no processo de viver e envelhecer dos idosos estudantes/integrantes. Revista Brasileira de Geriatria e Gerontologia, Rio de Janeiro: UnATI.Uerj, vol. 6, n.1, 2003.

SIMÃO, A, M. V.; FRISON, L. M. B. Autorregulação da aprendizagem: abordagens teóricas e desafios para as práticas em contextos. Cadernos de Educação UFPel, Pelotas: UFPel, vol. 45, n.2, p. 1- 20, 2013.

VIEIRA, Celia Maria de Souza Sanchez. Vida e morte: uma educação para a longevidade. Revista Memorialidades, Ilhéus: UESC, vol. 1 n.13, p. 73-94, 2010.

WILLCOX, D. C.; et al. Caloric restriction and human longevity: what can we learn from the Okinawans? Biogerontology, vol. 3, n.7, p.173-177, 2006. 
ENVELHECIMENTO ATIVO: UM PANORAMA DO INGRESSO DE IDOSOS NA UNIVERSIDADE

Data de recebimento: 10/10/2016

Data de aceite: 26/10/2016 